\title{
Erratum to: Fusion transcript loci share many genomic features with non-fusion loci
}

John Lai ${ }^{1,2,6}$, Jiyuan An ${ }^{1,2}$, Inge Seim ${ }^{1,2,3,4}$, Carina Walpole ${ }^{1,2}$, Andrea Hoffman ${ }^{1,2}$, Leire Moya ${ }^{1,2}$, Srilakshmi Srinivasan ${ }^{1,2}$, Joanna L. Perry-Keene ${ }^{5}$, Australian Prostate Cancer Bioresource, Chenwei Wang ${ }^{1,2}$, Melanie L. Lehman ${ }^{1,2}$, Colleen C. Nelson ${ }^{1,2}$, Judith A. Clements ${ }^{1,2}$ and Jyotsna Batra ${ }^{1,2^{*}}$ (i)

\section{Erratum}

After publication of the original article [1], a reader noted that one reference cited in the main text had not been mentioned in the References section. The reference (Qin et al., [2]) was cited as Ref. 33 within the text, but mistakenly did not appear in the References. As such the total number of References was also incorrect - there should have been 36 in total. References 33 - 35 should have been numbered $34-36$ in the main text and in the References section.

\footnotetext{
Author details

${ }^{1}$ Australian Prostate Cancer Research Centre - Queensland, Translational Research Institute, Brisbane, Australia. ${ }^{2}$ Cancer and Molecular Medicine Program, Institute of Health and Biomedical Innovation, Queensland University of Technology, Brisbane, Australia. ${ }^{3}$ Comparative and Endocrine Biology Laboratory, Institute of Health and Biomedical Innovation, Brisbane, Australia. ${ }^{4}$ Ghrelin Research Group, Institute of Health and Biomedical Innovation, Brisbane, Australia. ${ }^{5}$ Anatomical Pathology, Pathology Queensland, Brisbane, Australia. ${ }^{6}$ Current address: Genetic Technologies, 60-66 Hanover Street, Melbourne, Australia.
}

Published online: 03 June 2016

\section{References}

1. Lai J, An J, Seim I, Walpole C, Hoffman A, Moya L, et al. Fusion transcript loci share many genomic features with non-fusion loci. BMC Genomics. 2015;16: 1201. doi:10.1186/s12864-015-2235-4.

2. Qin F, Song Z, Babiceanu M, Song Y, Facemire L, et al. Discovery of CTCFsensitive Cis-spliced fusion RNAs between adjacent genes in human prostate cells. PLoS Genet. 2015;11(2):e1005001. doi:10.1371/journal.pgen. 1005001.

\footnotetext{
* Correspondence: jyotsna.batra@qut.edu.au

${ }^{1}$ Australian Prostate Cancer Research Centre - Queensland, Translational Research Institute, Brisbane, Australia

${ }^{2}$ Cancer and Molecular Medicine Program, Institute of Health and Biomedical Innovation, Queensland University of Technology, Brisbane, Australia
} 